\title{
盐酸黄连素的汇聚式合成研究
}

\author{
陈 程 ${ }^{a}$ 徐蒙蒙 ${ }^{a}$ 赵 青 $b$ 刘承秀 $c$ 杨鸿均*, $a$ 冯豫川*,a \\ ( ${ }^{a}$ 西南民族大学化学与环境保护工程学院 成都 610041) \\ $\left({ }^{b}\right.$ 西南民族大学预科教育学院 成都 610041) \\ ( ${ }^{c}$ 西南民族大学校医院 成都 610041)
}

\begin{abstract}
摘要 报道了以 1,2 -亚甲二氧基苯为起始原料合成盐酸黄连素的汇聚式路线，该路线包括格氏试剂亲核开环、脱保护、 环合等 5 步反应，总收率为 $33 \%$. 该路线反应条件温和、操作简单，且所有中间体及产物都经过 ${ }^{1} \mathrm{H} \mathrm{NMR、}{ }^{13} \mathrm{C} \mathrm{NMR、}$ MS 鉴定.

关键词 盐酸黄连素; 汇聚式合成; 1,2-亚甲二氧基苯; 格氏试剂
\end{abstract}

\section{A Concisely Convergent Synthesis of Berberine Chloride}

\author{
Chen, Cheng $^{a} \quad \mathrm{Xu}$, Mengmeng $^{a}$ \\ ${\text { Zhao, } \text { Qing }^{b} \quad \text { Liu, Chengxiu }}^{c}$ \\ Yang, Hongjun*,a \\ Feng, Yuchuan ${ }^{*, a}$ \\ $\left({ }^{a}\right.$ College of Chemistry \& Environment Protection Engineering, Southwest University for Nationalities, Chengdu 610041) \\ ( ${ }^{b}$ Preparatory School, Southwest University for Nationalities, Chengdu 610041) \\ ( ${ }^{c}$ Southwest University for Nationalities Hospital, Chengdu 610041)
}

\begin{abstract}
The berberine chloride was synthesized starting from benzo $[d][1,3]$ dioxole with the mild condition and the simple operation in overall $33 \%$ yield. The route includes the five steps of nucleophilic ring opening, deprotection and cyclization and so on. All intermediates were determined by ${ }^{1} \mathrm{H}$ NMR, ${ }^{13} \mathrm{C}$ NMR and MS techniques.
\end{abstract}

Keywords berberine chloride; convergent synthesis; benzo[ $d][1,3]$ dioxole; grignard regent

盐酸黄连素是存在于植物中的异喹啉类生物碱, 可 从多种植物中提取, 如黄连、黄柏、三颗针、伏牛花、 白屈菜、南天竹、小檗、铁皮莲等 ${ }^{[1 a]}$. 大量临床研究表 明, 盐酸黄连素具有抗癌 ${ }^{[1]} 、$ 抗炎 ${ }^{[2] 、}$ 抗微生物 ${ }^{[3]}$ 、抗病 毒 $^{[5]}$ 、抗利什曼原虫 ${ }^{[6]}$ 、抗寄生虫 ${ }^{[7]}$ 、抗结核 ${ }^{[8,9]}$ 等多种 药理活性, 而且其毒性低、副作用小, 市场需求大. 但可 供提取的天然植物资源有限. 因此, 人们致力于研究盐 酸黄连素及其类似物的化学合成方法 ${ }^{[8]}$.

据文献报道, 盐酸黄连素的合成大多是以 3,4-亚甲 二氧基苯乙胺 (2) 作关键中间体, 且主要有四种合成策 略(图 1). 1913 年, Decker 等课题组 ${ }^{[8]}$ 通过霍夫曼反应合 成化合物 2, 该路线具有原料不易得、合成收率低等不 足(路线 I); 南宁制药厂 ${ }^{[8 c]}$ 以胡椒醛为起始原料, 采用
Henry 反应、催化加氢等合成化合物 $\mathbf{2}$; 杭州制药厂[8e] 通过胡椒苄氯与氧化钠亲核取代反应，再经过还原而得 化合物 2(路线 II), 尽管收率较高但需使用氧化钠(路线 $\mathrm{III}$; 东北制药厂 ${ }^{[8 f]}$ 以苯酚为起始原料, 经过亚甲基化, 水解等 8 步合成盐酸黄连素(路线 IV). 尽管盐酸黄连素 已经工业化生产, 但现有的路线仍然需要深入地研究和 改进, 从而开发出一条反应条件温和、路线简短的新路 线.

受到以上研究的启发, 2016 年我们课题组 ${ }^{[8 n]}$ 对霍夫 曼路线进行了改进, 开发了盐酸黄连素新的合成路线. 同时我们也发现，合成盐酸黄连素都是采用直线式逐步 合成的策略，效率较低、路线长. 因此，我们设想开发效 率更高，路线更短的汇聚式合成路线(Scheme 1). 通过

\footnotetext{
* Corresponding author. E-mail: yanghj@swun.cn

Received December 2, 2016; revised January 1, 2017; published online January 17, 2017.

Project supported by the Graduate Innovative Research Projects of Central Universities, Southwest University for Nationalities (No. CX2015SZ045) and the Research Projects Sichuan Provincial Department of Education (No. 15ZB0487).

西南民族大学研究生创新(No. CX2015SZ045)和四川省教育厅(No. 15ZB0487)资助项目.
} 


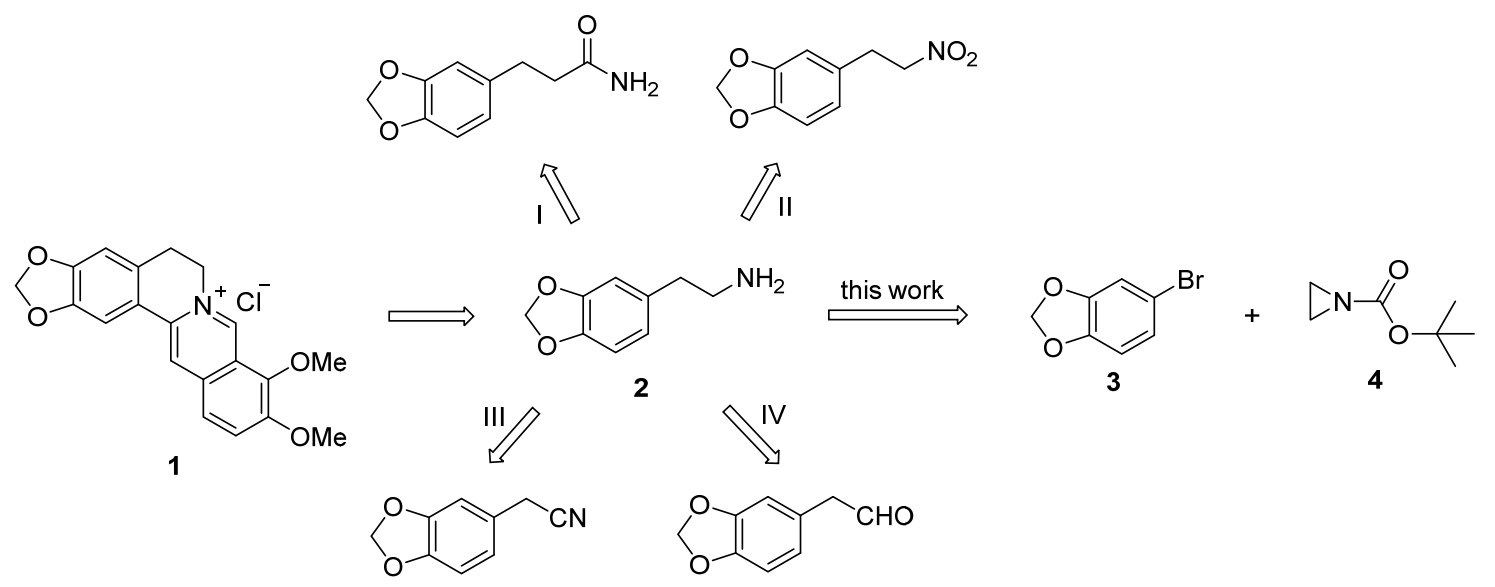

图 1 盐酸黄连素的逆合成分析

Figure 1 Retrosynthesis analysis of berberine chloride

对盐酸黄连素的逆合成分析, 盐酸黄连素可通过化合物 $\mathbf{2}$ 合成得到，化合物 $\mathbf{2}$ 可通过 $\mathbf{3}$ 和 $\mathbf{4}$ 反应得到. 基于逆合 成分析, 本文以 1,2-亚甲二氧基苯为起始原料, 经溴化 反应得化合物 $\mathbf{4}$, 化合物 5 经二碳酸二叔丁酯保护得化 合物 3. 在化合物 $\mathbf{4}$ 的格氏试剂作用下化合物 $\mathbf{3}$ 亲核开 环得 7, 再脱叔丁氧羰基即得关键中间体 2 . 中间体 2 再 经缩合、环化得盐酸黄连素, 该汇聚式合成路线仅需 5 步，收率高达 $33 \%$.

\section{1 结果与讨论}

\section{1 化合物 4 的合成}

在化合物 $\mathbf{6}$ 的溴代反应中, 最初我们尝试用溴化 氢一双氧水作为溴化试剂, 但副产物多, 收率仅为 $45 \%$. 以硝酸铈铵(CAN)-溴化锂作为溴化试剂, 收率可提高 至 $77 \%$, 反应条件温和, 因而选 CAN-溴化锂为溴化试 剂合成化合物 4. CAN 作为单电子氧化剂, 将 $\mathrm{Br}^{-}$氧化为
$\mathrm{Br}^{+}$, 进而 $\mathrm{Br}^{+}$进攻富电子的芳环发生亲电取代, 得到化 合物 $\mathbf{4}^{[9]}$ (Scheme 2).

\section{2 化合物 7 的合成}

在格氏试剂的作用下，化合物 3 可开环得化合物 7. 为了提高收率, 我们对这一步反应进行条件研究, 考察 了催化剂、温度、溶剂对收率的影响(表 1). 实验结果表 明， $-40{ }^{\circ} \mathrm{C}$ 时，以四氢呋喃(THF)作溶剂，格氏试剂直 接与 3 反应主要生成化合物 $\mathbf{9}$, 并没有得到中间体 7 (表 1, Entry 1); 当以 $\mathrm{CuI}$ 作催化剂, 尽管能得到中间体 7, 但收率仅为 $7 \%$ (表 1, Entry 2); 改用 $\mathrm{CuBr}$ 作为催化剂 时, 收率略微提高(表 1, Entry 3); 以 $\mathrm{CuBr} \cdot \mathrm{SMe}_{2}$ 作催化 剂, 收率可提高至 $34 \%$ (表 1, Entry 4). 为了进一步提高 收率, 探讨温度对反应的影响, 随着温度的提高, 收率 逐渐增加, 在 $0{ }^{\circ} \mathrm{C}$ 时收率可达 $78 \%$, 但继续升高温度, 收率急剧下降(表 1, Entry 7). 在 $0{ }^{\circ} \mathrm{C}$, 以乙醚作溶剂时, 格氏试剂很难生成(表 1, Entry 8). 因此, 合成化合物 7

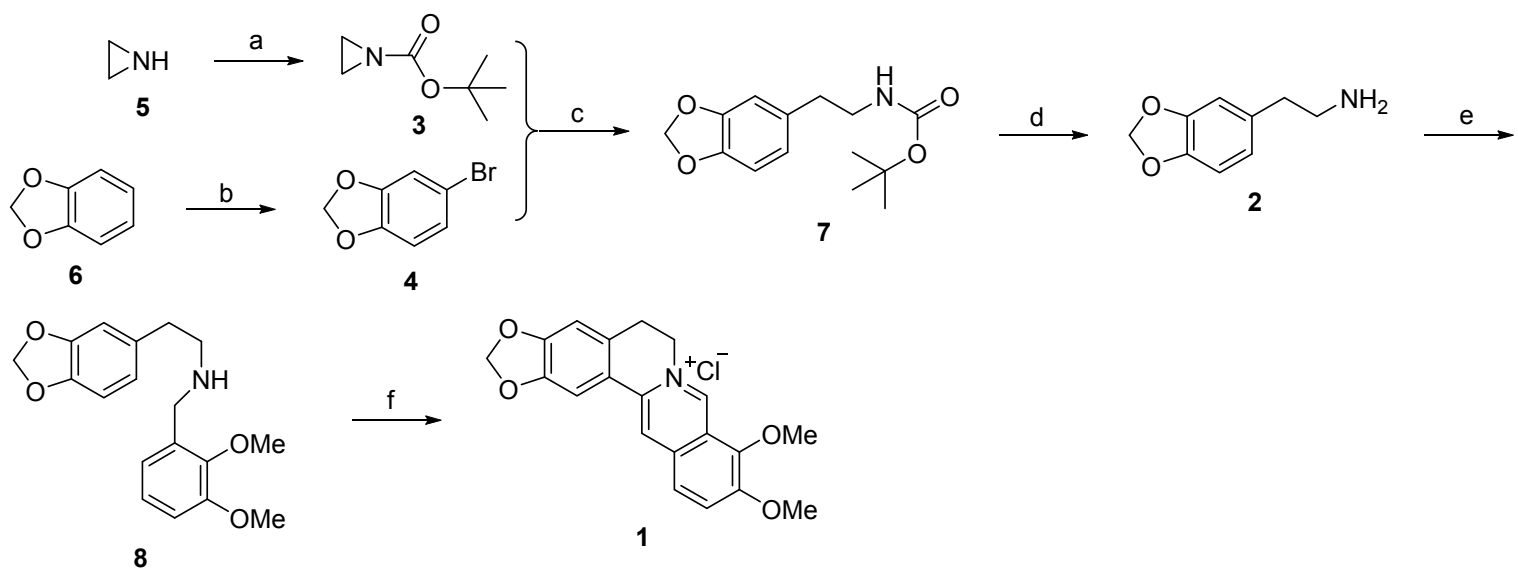

Reagents and conditions: (a) (Boc) ${ }_{2} \mathrm{O}, \mathrm{Et}_{3} \mathrm{~N}, \mathrm{CH}_{2} \mathrm{Cl}_{2}$, r.t., $4 \mathrm{~h}, 93 \%$; (b) $\mathrm{CAN}, \mathrm{LiBr}, \mathrm{CH}_{3} \mathrm{CN}, 32{ }^{\circ} \mathrm{C}, 8 \mathrm{~h}, 77 \%$; (c) $\mathrm{Mg}, \mathrm{I}_{2}, \mathrm{THF} \mathrm{CuBr} \cdot \mathrm{SMe}{ }_{2}, 0{ }^{\circ} \mathrm{C}$, 20 min, 78 \%; (d) TFA, $\mathrm{CH}_{2} \mathrm{Cl}_{2}$, r.t., 17 h, $94 \%$; (e) 2,3-dimethoxybenzaldehyde, $\mathrm{CH}_{3} \mathrm{OH}, \mathrm{NaBH}_{4}, 70 \sim 105{ }^{\circ} \mathrm{C}, 10.5$ h, $96 \%$; (f) $\mathrm{HCOOH}$, $\mathrm{CHOCHO}, \mathrm{CuSO}_{4}, 60{ }^{\circ} \mathrm{C}, 4.5 \mathrm{~h}, 60 \%$

图式 1 盐酸黄连素的汇聚式合成

Scheme 1 Convergent synthesis of berberine chloride 
$2 \mathrm{Ce}^{4+}+\mathrm{Br}^{-} \stackrel{\text { 氧化 }}{\longrightarrow} \mathrm{Br}^{+}+2 \mathrm{Ce}^{3+}$

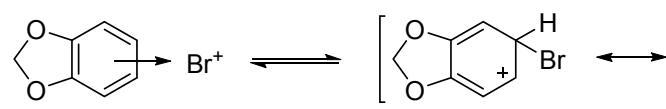

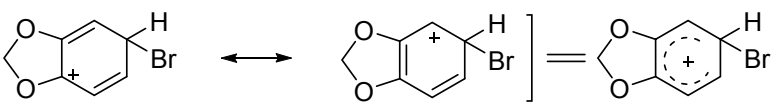<smiles>CCCCCCC</smiles>

图式 2 合成化合物 $\mathbf{4}$ 的反应机理

Scheme 2 Reaction mechanism on the preparation of 4

表 1 合成化合物 7 的反应条件优化

Table 1 Optimization study on the preparation of 7<smiles>CC(C)(C)OC(=O)N1C[C@H]1CC(=O)OC(F)(F)F</smiles><smiles>CC(C)(C)OC(=O)NCCc1ccc2c(c1)OCO2</smiles><smiles>CC1CN1C(=O)c1ccc2c(c1)OCO2</smiles>

9

\begin{tabular}{clrcc}
\hline Entry & Catalyst & $t /^{\circ} \mathrm{C}$ & Solvent $/ \mathrm{mL}$ & Yield $^{a} / \%$ \\
\hline 1 & - & -40 & THF & - \\
2 & $\mathrm{CuI}$ & -40 & $\mathrm{THF}$ & 7 \\
3 & $\mathrm{CuBr}$ & -40 & $\mathrm{THF}$ & 23 \\
4 & $\mathrm{CuBr} \bullet \mathrm{SMe}_{2}$ & -40 & $\mathrm{THF}$ & 34 \\
5 & $\mathrm{CuBr} \bullet \mathrm{SMe}_{2}$ & -20 & $\mathrm{THF}$ & 43 \\
6 & $\mathrm{CuBr} \bullet \mathrm{SMe}_{2}$ & 0 & $\mathrm{THF}$ & 78 \\
7 & $\mathrm{CuBr} \bullet \mathrm{SMe}_{2}$ & 30 & $\mathrm{THF}$ & 28 \\
8 & $\mathrm{CuBr} \bullet \mathrm{SMe}_{2}$ & 0 & $\mathrm{Et}_{2} \mathrm{O}$ & - \\
\hline
\end{tabular}

a 纯化的收率.

的最佳反应条件为: 干燥 $\mathrm{THF}$ 为溶剂, $\mathrm{CuBr} \cdot \mathrm{SMe}_{2}$ 为反 应催化剂, 反应温度为 $0{ }^{\circ} \mathrm{C}$, 在该反应条件下, 合成化 合物 7 的收率为 78\%(表 1, Entry 7).

\section{3 化合物 7 的合成反应机理推测}

根据实验反应的现象和结果，对合成化合物 7 的反 应机理进行预测(Scheme 3). 当反应中无催化剂时, 格 氏试剂直接进攻羰基碳原子而生成化合物 9; 当加入催 化剂 $\mathrm{CuBr} \cdot \mathrm{SMe}_{2}$ 时, 首先 $\mathrm{CuBr} \bullet \mathrm{SMe}_{2}$ 与格氏试剂形成芳 基铜络合物, 可能由于铜络合物位阻较大，选择性地进 攻位阻较小的氮杂环丙烷碳原子, 开环得到目标化合物 7.

\section{2 结论}

本文开发了盐酸黄连素的汇聚式合成路线, 以 1,2亚甲二氧基苯为起始原料，经格氏试剂的亲核开环作为 本路线的关键步骤，仅需 5 步反应合成盐酸黄连素，收 率为 $33 \%$. 所有中间体都经核磁氢谱、碳谱、质谱，红 外和熔点鉴定. 该路线具有路线短、收率高、反应条件 温和等特点, 为合成盐酸黄连素提供一种新思路新途 径，具有潜在的工业应用价值.

\section{3 实验部分}

\section{1 仪器与试剂}

${ }^{1} \mathrm{H}$ NMR、 ${ }^{13} \mathrm{C}$ NMR 用 Agilent-400 (400 MHz)型核 磁共振仪测定, TMS 作内标, $\mathrm{CDCl}_{3}$ 或 $d_{6}$-DMSO 作溶 剂; 质谱用 LCQ ADVANTAGE MAX 测定; 熔点用 WRS-1B数字熔点仪测定; 红外用FTS 3000 傅里叶变换 红外光谱仪(美国 DIGILAB 公司)测定. 其余试剂均为国 产分析纯试剂，四氢呋喃和二氯甲烷干燥后使用，其余 试剂未经纯化直接使用.

\section{2 实验方法}

\subsubsection{5-溴苯并 $[d][1,3]$ 二氧杂环戊烯 $(4)$ 的合成}

在 $\mathrm{N}_{2}$ 保护下, 将化合物 $6(20.0 \mathrm{~g}, 164 \mathrm{mmol})$ 、无水 溴化锂 $(15.7 \mathrm{~g}, 181 \mathrm{mmol})$ 和 $100 \mathrm{~mL}$ 乙腈依次加入 1000<smiles>CCC(C)CC1CN1C(=O)OC(C)(C)C</smiles>

图式 3 化合物 7 的合成反应机理推测

Scheme 3 Proposed reaction mechanism of 7 
$\mathrm{mL}$ 三口瓶中. 将反应液温度升至 $32{ }^{\circ} \mathrm{C}$, 摚拌下滴加硝 酸铈铵 $(99.0 \mathrm{~g}, 181 \mathrm{mmol})$ 的乙腈 $(400 \mathrm{~mL})$ 溶液, 薄层色 谱(TLC)跟踪反应完全, 冷却至室温, 加入 $100 \mathrm{~mL} \mathrm{H}_{2} \mathrm{O}$, 乙醚萃取 $(300 \mathrm{~mL} \times 3)$, 合并有机相, 再依次用饱和 $\mathrm{NaHCO}_{3}(80 \mathrm{~mL} \times 3), \mathrm{H}_{2} \mathrm{O}(80 \mathrm{~mL} \times 3)$, 饱和食盐水洗 涤, 最后经无水 $\mathrm{Na}_{2} \mathrm{SO}_{4}$ 干燥得初产品, 经减压蒸馏收集 $120 \sim 128{ }^{\circ} \mathrm{C}(0.095 \mathrm{MPa})$ 的馏分, 得 $25.3 \mathrm{~g}$ 浅黄色油状 液体 $\mathbf{4}^{[9 \mathrm{a}]}$, 收率为 $77 \%$. ${ }^{1} \mathrm{H}$ NMR $\left(400 \mathrm{MHz}^{\circ} \mathrm{CDCl}_{3}\right) \delta$ : 5.96 (s, 2H), 6.68 (d, $J=4.0 \mathrm{~Hz}, 1 \mathrm{H}), 6.94$ (dd, $J=4.4 \mathrm{~Hz}$, $2 \mathrm{H}) ;{ }^{13} \mathrm{C}$ NMR $\left(100 \mathrm{MHz}, \mathrm{CDCl}_{3}\right) \delta: 101.5,109.5,112.2$, 113.0, 124.2, 146.9, 148.5; IR (KBr) v: 2907, 1615, 1483, 1239, 1158, 1109, 1043, 937, $866 \mathrm{~cm}^{-1}$; ESI-MS m/z (\%): $200.71[\mathrm{M}+\mathrm{H}]^{+}$.

\subsection{2 叔丁基氮丙啶-1-羧酸酯(3)的合成}

将氮丙啶 $(1.00 \mathrm{~g}, 23.3 \mathrm{mmol}) 、 20 \mathrm{~mL}$ 二氯甲烷和 9.6 $\mathrm{mL}$ 三乙胺依次加入 $100 \mathrm{~mL}$ 三口瓶中, 于 $0{ }^{\circ} \mathrm{C}$ 下, 搅拌 下滴加二碳酸二叔丁酯 $(5.03 \mathrm{~g}, 23.3 \mathrm{mmol})$ 的二氯甲烷 $(20 \mathrm{~mL})$ 溶液, 室温下搅拌反应, TLC 跟踪反应完全, 冷 却至室温, 在旋转蒸发仪上蒸去溶剂, 得到的残余液用 中性 $\mathrm{Al}_{2} \mathrm{O}_{3}$ (石油醚)纯化, 得 $3.10 \mathrm{~g}$ 无色油状液体 3, 收 率为 93\%. ${ }^{1} \mathrm{H}$ NMR $\left(400 \mathrm{MHz}, \mathrm{CDCl}_{3}\right) \delta: 1.42(\mathrm{~s}, 9 \mathrm{H})$, $2.10(\mathrm{~s}, 4 \mathrm{H}) ;{ }^{13} \mathrm{C} \mathrm{NMR}\left(100 \mathrm{MHz}, \mathrm{CDCl}_{3}\right) \delta: 25.71,27.84$, 81.11, 162.76; IR (KBr) v: 2992, 2934, 1747, 1396, 1343, 1230, 1176, $1052 \mathrm{~cm}^{-1}$; ESI-MS m/z (\%): $144.01[\mathrm{M}+$ $\mathrm{H}]^{+}$.

3.2 .3 [2-(苯并 $[d][1,3]$ 二氧杂环戊烯-5-基)乙基]氨基 甲酸叔丁酯(7)的合成

在 $\mathrm{N}_{2}$ 保护下, 将镁条 $\left(134 \mathrm{mg}, 2.82 \mathrm{mmol}\right.$ ) 和 $\mathrm{I}_{2}$ 依次 加入 $25 \mathrm{~mL}$ 三口瓶, 往反应瓶中先加入一部分化合物 4 (1.02 g, $5.12 \mathrm{mmol})$ 的无水四氢呋喃(THF) $(8 \mathrm{~mL})$ 溶液, 格氏反应引发完毕后缓慢加入剩余溶液, 制成格氏试 剂, 以备使用. 将 $\mathrm{CuBr} \bullet \mathrm{SMe}_{2}(0.0421 \mathrm{mg}, 0.205 \mathrm{mmol})$ 用 $4 \mathrm{~mL}$ 的 THF 溶解, 在 $0{ }^{\circ} \mathrm{C}$ 下, 用注射器将制备好的 格氏试剂加入到 $\mathrm{CuBr} \cdot \mathrm{SMe}_{2}$ 的反应液中, 摚拌反应 5 $\mathrm{min}$, 后滴加化合物 3 (400 mg, $2.80 \mathrm{mmol}$ ) 的无水 THF (4 $\mathrm{mL}$ ) 溶液, 搅拌反应 $20 \mathrm{~min}$, 停止反应. 加入 $20 \mathrm{~mL}$ 的饱 和 $\mathrm{NH}_{4} \mathrm{Cl}$ 溶液，二氯甲烷萃取 $(50 \mathrm{~mL} \times 3)$, 合并有机相， 饱和食盐水洗涤, 最后经无水 $\mathrm{Na}_{2} \mathrm{SO}_{4}$ 干燥得初产品, 经 柱层析 $[V$ (石油醚 $): V($ 二氧甲烷 $)=1: 10]$ 纯化后, 得 $576 \mathrm{mg}$ 白色固体结晶 7, 收率为 78\%. m.p. 59 61 ${ }^{\circ} \mathrm{C}$ (文献值 ${ }^{[10]}: 60{ }^{\circ} \mathrm{C}$ ); ${ }^{1} \mathrm{H}$ NMR $\left(400 \mathrm{MHz}, \mathrm{CDCl}_{3}\right) \delta: 1.42$ (s, 9H), 2.69 (t, $J=6.6 \mathrm{~Hz}, 2 \mathrm{H}), 3.30(\mathrm{~s}, 2 \mathrm{H}), 4.56(\mathrm{~s}, 1 \mathrm{H})$, $5.91(\mathrm{~s}, 2 \mathrm{H}), 6.66 \sim 6.61(\mathrm{~m}, 2 \mathrm{H}), 6.73(\mathrm{dd}, 1 \mathrm{H}) ;{ }^{13} \mathrm{C} \mathrm{NMR}$ $\left(100 \mathrm{MHz}, \mathrm{CDCl}_{3}\right) \delta: 28.37,35.86,41.93,79.19,100.82$, $108.28,109.08,121.62,132.72,146.05,147.69,155.81$; IR
(KBr) v: 3375, 2991, 2901, 1689, 1535, 1500, 1451, 1374, 1254, 1181, 1044, 936, 876, 811, $629 \mathrm{~cm}^{-1}$; ESI-MS $\mathrm{m} / z$ (\%): $266.21[\mathrm{M}+\mathrm{H}]^{+}$.

\subsubsection{3,4-亚甲二氧基苯乙胺(2)的合成}

将化合物 7 (200 mg, $0.755 \mathrm{mmol})$ 和 $7 \mathrm{~mL}$ 二氯甲烷 依次加入 $25 \mathrm{~mL}$ 的三口瓶中, $0{ }^{\circ} \mathrm{C}$ 下滴加三氟乙酸 (TFA) (714 mg, $6.27 \mathrm{mmol})$ 的二氯甲烷 $(2.5 \mathrm{~mL})$ 溶液, 在 室温条件下反应 $17 \mathrm{~h}$, 停止反应, 加入 $15 \mathrm{~mL} 2 \mathrm{~mol} / \mathrm{L}$ $\mathrm{HCl}$, 分出水相, 调 $\mathrm{pH}=10.0$, 用二氯甲烷萃取 $(30$ $\mathrm{mL} \times 3)$, 合并有机相，饱和食盐水洗涤，最后经无水 $\mathrm{Na}_{2} \mathrm{SO}_{4}$ 干燥, 得 $116 \mathrm{mg}$ 浅黄色油状液体 2 , 收率为 $94 \%$. ${ }^{1} \mathrm{H}$ NMR (400 MHz, $\left.\mathrm{CDCl}_{3}\right) \delta: 2.66(\mathrm{t}, J=6.8 \mathrm{~Hz}, 2 \mathrm{H})$, $2.90(\mathrm{t}, J=6.8 \mathrm{~Hz}, 2 \mathrm{H}), 5.92(\mathrm{~s}, 2 \mathrm{H}), 6.64(\mathrm{~d}, J=7.6 \mathrm{~Hz}$, $1 \mathrm{H}), 6.69$ (s, 1H), $6.74(\mathrm{~d}, J=8.0 \mathrm{~Hz}, 1 \mathrm{H}) ;{ }^{13} \mathrm{C}$ NMR $(100$ $\left.\mathrm{MHz}, \mathrm{CDCl}_{3}\right) \delta: 39.6,43.6,100.8,108.2,109.1,121.6$, 133.5, 145.9, 147.6; IR (KBr) v: 3382, 2936, 1604, 1499, 1366, 1250, 1199, 1106, 1043, 933, 866, $813 \mathrm{~cm}^{-1}$; ESI-MS $m / z(\%): 166.16[\mathrm{M}+\mathrm{H}]^{+}$.

3.2.5 N-(2,3-二甲氧基芐基)-3,4-亚甲二氧基苯乙胺 (8) 的合成

将化合物 2 (3.00 g, $18.2 \mathrm{mmol}) 、 2,3$-二甲氧基苯甲 醛(3.01 g, $18.1 \mathrm{mmol})$ 依次加入三口瓶中, 在 $105{ }^{\circ} \mathrm{C}$ 条 件下反应 $0.5 \mathrm{~h}$, 随后冷却至室温, 加入 $24 \mathrm{~mL}$ 甲醇, 分 多次加入 $\mathrm{NaBH}_{4}(381 \mathrm{mg}, 10.0 \mathrm{mmol})$, 在 $70{ }^{\circ} \mathrm{C}$ 的条件 下反应 $10 \mathrm{~h}$. 室温下旋转蒸除去多余的甲醇, 残留物用 $20 \mathrm{~mL}$ 水溶解后用乙醚萃取 $(50 \mathrm{~mL} \times 3)$, 合并有机层, 用 $20 \mathrm{~mL}$ 水洗、饱和食盐水洗 $(20 \mathrm{~mL} \times 2)$, 有机相用无 水 $\mathrm{Na}_{2} \mathrm{SO}_{4}$ 干燥，过滤洗涤，合并滤液，旋干，得 $5.48 \mathrm{~g}$ 淡黄色油状液体 $8^{[8 \mathrm{e}]}$, 收率 $96 \%$. ${ }^{1} \mathrm{H} \mathrm{NMR}(400 \mathrm{MHz}$, $\left.\mathrm{CDCl}_{3}\right) \delta: 2.52(\mathrm{~s}, 2 \mathrm{H}), 2.75(\mathrm{t}, J=7.2 \mathrm{~Hz}, 2 \mathrm{H}), 2.84(\mathrm{t}$, $J=6.8 \mathrm{~Hz}, 2 \mathrm{H}), 3.80(\mathrm{~s}, 3 \mathrm{H}), 3.85(\mathrm{~s}, 3 \mathrm{H}), 5.90(\mathrm{~s}, 2 \mathrm{H})$, $6.62 \sim 6.72(\mathrm{~m}, 3 \mathrm{H}), 6.83 \sim 6.86(\mathrm{~m}, 2 \mathrm{H}), 7.00(\mathrm{t}, J=8.0$ $\mathrm{Hz}, 1 \mathrm{H}) ;{ }^{13} \mathrm{C} \mathrm{NMR}\left(\mathrm{CDCl}_{3}, 100 \mathrm{MHz}\right) \delta:{ }^{13} \mathrm{C}$ NMR $(100$ $\left.\mathrm{MHz}, \mathrm{CDCl}_{3}\right) \delta: 35.8,48.4,50.4,55.6,60.6,100.7,108.1$, $109.0,111.4,121.5,121.7,123.8,133.2,133.6,145.8$, 147.2, 147.5, 152.5; IR (KBr) v: 3341, 2947, 2851, 1597, 1494, 1453, 1360, 1248, 1049, 934, 865, $808 \mathrm{~cm}^{-1}$; ESI-MS $m / z: 316.21\left([\mathrm{M}+\mathrm{H}]^{+}\right)$.

3.2.6 5,6-二氢-9,10-二甲氧基苯并 $(g) 1,3$-苯并二恶 茂 $(5,6-a)$ 喹嗪盐酸盐 $(1)$ 的合成

将 $6.6 \mathrm{~mL} 88 \%$ 甲酸、硫酸铜 $(1.23 \mathrm{~g}, 7.71 \mathrm{mmol})$ 依 次加入反应瓶中, 升温至 $55{ }^{\circ} \mathrm{C}$ 搅拌反应, 保温脱水 0.5 $\mathrm{h}$, 再加入化合物 8 (1.32 g, $4.19 \mathrm{mmol}) 、 1.1 \mathrm{~mL} 40 \%$ 乙 二醛溶液, 升温至 $100{ }^{\circ} \mathrm{C}$ 反应 $4 \mathrm{~h}$. 在反应过程中按下 列比例加入浓盐酸, 保温后 $50 \mathrm{~min}$, 加入 $0.1 \mathrm{~mL}$ 浓盐 
酸, 隔 $50 \mathrm{~min}$ 后, 加入 $0.1 \mathrm{~mL}$ 浓盐酸, 再隔 $1 \mathrm{~h}$ 后, 加 入 $0.1 \mathrm{~mL}$ 浓盐酸, 再隔 $1 \mathrm{~h}$, 加入 $0.1 \mathrm{~mL}$ 浓盐酸, 再保 温反应 $20 \mathrm{~min}$. 搅拌冷却至 $0{ }^{\circ} \mathrm{C}$ 以下, 过滤, 用 $5 \%$ 食盐 水洗至 $\mathrm{pH}=2.0$, 过滤抽干得盐酸黄连素粗品. 将盐酸 黄连素粗品先加部分水调成浆状, 然后加水至足量, 升 温至 $75 \sim 80{ }^{\circ} \mathrm{C}$, 保温 $1 \mathrm{~h}$, 然后加入氧化钙调至 $\mathrm{pH}=$ $8.0 \sim 8.5$ 趁热过滤, 滤饼用热水洗涤, 洗液与滤液合并 后用 $10 \%$ 盐酸调至 $\mathrm{pH}=2.0$, 室温放置 $8 \mathrm{~h}$, 过滤, 滤饼 用常水洗涤, 抽干、干燥, 得一次精制盐酸黄连素, 得 $839 \mathrm{mg}$ 黄色固体 $\mathbf{1}^{[11]}$, 收率 $60 \%$. m.p. 195 $197{ }^{\circ} \mathrm{C}$ (文 献值 $\left.{ }^{[8 \mathrm{n}]} 195 \sim 197{ }^{\circ} \mathrm{C}\right)$; ${ }^{1} \mathrm{H}$ NMR (400 MHz, DMSO- $\left.d_{6}\right) \delta$ : $3.19 \sim 3.22(\mathrm{t}, J=5.8 \mathrm{~Hz}, 2 \mathrm{H}), 4.07(\mathrm{~s}, 3 \mathrm{H}), 4.10(\mathrm{~s}, 3 \mathrm{H})$, $4.88 \sim 4.91(\mathrm{t}, J=6 \mathrm{~Hz}, 2 \mathrm{H}), 6.18(\mathrm{~s}, 2 \mathrm{H}), 7.09(\mathrm{~s}, 1 \mathrm{H})$, 7.80 (s, 1H), 7.99 8.01 (d, $J=8.8 \mathrm{~Hz}, 1 \mathrm{H}), 8.20 \sim 8.22$ (d, $J=8.8 \mathrm{~Hz}, 1 \mathrm{H}), 8.94$ (s, 1H), 9.89 (s, 1H); ${ }^{13} \mathrm{C}$ NMR $(100$ MHz, DMSO- $\left.d_{6}\right) \delta: 26.7,55.5,57.4,62.3,102.5,105.8$, $108.8,120.6,120.8,121.8,123.9,127.0,131.0,133.3$, 137.8, 144.0, 145.8, 148.0, 150.1, 150.8; IR (KBr) v: 3424, 3071, 2925, 1612, 1510, 1393, 1343, 1278, 1232, 1110, $1043 \mathrm{~cm}^{-1}$; ESI-MS m/z (\%): $337.18[\mathrm{M}+\mathrm{H}]^{+}$.

辅助材料(Supporting Information) 产物和中间体的 核磁共振和质谱图谱. 这些材料可以免费从本刊网站 (http://sioc-journal.cn/)上下载.

\section{References}

[1] (a) Lo, C. Y.; Hsu, L. C.; Chen, M. S.; Lin, Y. J.; Chen, L. G.; Kuo, C. D.; Wu., J. Y. Bioorg. Med. Chem. Lett. 2013, 23, 305. (b) Li, Q.; Xiang, J. F.; Tang, Y. L. Chin. J. Chem. 2015, 33, 1041.

[2] Zhang, S. L.; Chang, J. J.; Damu, G. V.; Fang, B.; Zhou, X. D.; Geng, R. X.; Zhou, C. H. Bioorg. Med. Chem. Lett. 2013, 23, 1008.

[3] (a) Samosorn, S.; Tanwirat, B.; Muhamad, N.; Casadei, G.; Tomkiewicz, D.; Lewis, K.; Suksamrarn, A.; Prammananan, T.; Gornall, K. C.; Beck, J. L.; Bremner, J. B. Bioorg. Med. Chem. 2009, 17, 3866.

(b) Zhang, S. L.; Damu, G. L.; Zhang, L.; Geng, R. X.; Zhou, C. H. Eur. J. Med. Chem. 2012, 55, 164.

[4] Bodiwala, H. S.; Sabde, S.; Mitra, D.; Bhutani, K. K.; Singh, I. P. Eur. J. Med. Chem. 2011, 46, 1045.
[5] Vennerstrom, J. L.; Lovelace, J. K.; Wsits, V. B.; Hanson, W. L.; Klayman, D. L. Antimicrob. Agents Chemother. 1990, 34, 918.

[6] Bahar, M.; Deng, Y.; Zhu, X.; He, S.; Pandharkar, T.; Drew, M. E.; Navarro-Vazquez, A.; Anklin, C.; Gil, R. R.; Dos Kotch, R. W.; Werbovetz, K. A.; Kinghorn, A. D. Bioorg. Med. Chem. Lett. 2011, $21,2606$.

[7] (a) Letasiova, S.; Jantova, S.; Cipak, L.; Muckova, M. Cancer Lett. 2006, 239, 254.

(b) Ma, Y.; Ou, T. M.; Tan, J. H.; Hou, J. Q.; Huang, S. L.; Gu, L. Q.; Huang, Z. S. Bioorg. Med. Chem. Lett. 2009, 19, 3414.

[8] (a) Decker, V. I. Justus Liebigs Ann. Chem. 1913, 395, 295.

(b) Kametani, T.; Noguchi, 1. J. Chem. Soc. (C) 1969, 2036.

(c) Nanning Pharmaceutical Chin. J. Pharm. 1973, (7), 1 (in Chinese).

(广西南宁制药厂，医药工业, 1973, (7), 1.)

(d) Masayuki, O.; Kumiko, Y.; Junko, O. Chem. Pharm. Bull. 1974, 22, 2365.

(e) Hangzhou First Pharmaceutical Reagent Chamber. Chin. J. Pharm. 1974, (8), 6 (in Chinese).

(杭州第一制药厂试剂室，医药工业， 1974, (8), 6.)

(f) North-east Pharmaceutical Factory Chin. J. Pharm. 1975, (4), 12 (in Chinese).

(东北制药总厂，医药工业, 1975, (4), 12.)

(g) Vinogradova, V. I.; Yunusov, M. S.; Kuchin, A. V.; Tolstikov, G. A.; Sagandykov, R. T.; Khaimuratov, K. A.; Ali mov, A. Chem. Nat. Compd. 1990, 26, 54.

(h) Hisashi, I.; Mayumi, O.; Shuji, O.; Takashi, H.; Tsutomu, I. Heterocycles 1994, 37, 897.

(i) Matulenko, M. A.; Meyers, A. I. J. Org. Chem. 1996, 61, 573.

(j) Yang, P.; Song, D. Q.; Li, Y. H.; Kong, W. J.; Wang, Y. X.; Gao, L. M.; Liu, S. Y.; Cao, R. Q.; Jiang, J. D. Bioorg. Med. Chem. Lett. 2008, $18,4675$.

(k) Gatland, A. E.; Pilgrim, B. S.; Procopiou, P. A.; Donohoe, T. J. Angew. Chem. Int. Ed. 2014, 53, 14555.

(1) He, Y.; Zheng, Y.; Hai, L.; Wu, Y. Chin. J. Chem. 2014, 32, 1121.

(m) Reddy, V.; Jadhav, A. S.; Vijaya Anand, R. Org. Biomol. Chem. 2015, 13, 3732.

(n) Chen, C.; Luo, Z. M.; Yang, H. J.; Feng, Y. C. Chin. J. Org. Chem. 2016, 36, 1426 (in Chinese).

(陈程, 罗卓玛, 杨鸿均, 冯豫川, 有机化学, 2016, 36, 1426.)

[9] (a) Roy, S. C.; Guin, C.; Rana, K. K.; Maiti, G. Tetrahedron Lett. 2001, 42, 6941.

(b) Roy, S. C.; Guin, C.; Maiti, G. Tetrahedron Lett. 2001, 42, 9253.

[10] Tietze, L. F., Schirok, H. J. Am. Chem. Soc. 1999, 121, 10264.

[11] Pan, J. F.; Yu, C.; Zhu, D. Y.; Zhang, H.; Ren, J. Y. CN 1314347, 2001 [Chem. Abstr. 2002, 137, 370266].

(Li, L.; Fan, Y.) 